\title{
Fluorescence studies on the 3d-ionization threshold range in krypton
}

\author{
M. Wildberger ${ }^{\text {a }}$, A. Ehresmann ${ }^{\text {a }}$, H. Schmoranzer ${ }^{\text {a }}$, B. Möbus ${ }^{\text {b }}$, B. Magel $^{\text {b }}$ \\ K.-H. Schartner ${ }^{b}$ \\ a Fachbereich Physik der Universität Kaiserslautern, Erwin Schrödinger Straße. D-67663 Kaiserslautern. German!̣. \\ b I. Physikalisches Institut der Justus-Liehig-L'niversität. Heinrich Buff Ring, D-35392 Giessen. Germany:
}

Received 11 May 1994; revised manuscript received 8 August 1994: accepted for publication 16 August 1994. Communicated by B. Frickc

\begin{abstract}
Krypton atoms were excited by photons in the energy range from the threshold for photoionization of the 3d-electrons up to $120 \mathrm{eV}$. and the fluorescence radiation in the spectral range from 780 to $965 \mathrm{~A}$ was observed and analyzed. Cross sections for the population of excited states in KrIll with at least one 4s-hole resulting from an Auger transition as the first decay step and for Krll satellites were determined. The energy dependence of the $3 \mathrm{~d}$-ionization cross section in the $3 \mathrm{~d}_{5 / 2}-$ and the $3 \mathrm{~d}_{3 / 2}$-threshold range was derived from the experimental data. The cross sections for production of KrIl states were found to follow the energy dependence of the 3d-cross sections.
\end{abstract}

\section{Introduction}

Up to now various experimental methods have been used to study the threshold range of photoionization of the 3d-electrons in atomic krypton. After the first energy-loss measurements with fast clectrons [1.2], monochromatized synchrotron radiation combined with different detection methods was employed. lon-yield measurements in the energy range of the $3 \mathrm{~d}^{-1} n \mathrm{p}$-resonances and $\mathrm{a}$ few $\mathrm{eV}$ above the ionization thresholds [3] led to absolute cross sections for the formation of singly, doubly and triply charged krypton ions. The first analysis of the kinetic energy of the electrons emitted on a resonance was carried out for the $3 d_{5 / 2}^{-1} 5 p$ - and the $3 d_{3 / 2}^{-1} 5 p$-resonances [4]. Later on more detailed investigations of electron energies were carried out [5-11] on the resonances. The application of electron coincidence techniques to measurements on the $3 d_{5 / 2}^{-1} 5 p$-resonance [12] rendered possible the quantitative observation of two- step decay processes, with a spectator or shake-up Auger decay in a first step and, in a second step. an autoionization decay to states in the doubly charged Ion. By analyzing the emission of threshold electrons in coincidence with ion signals [13], it was found that these electrons were not only produced in a single-step decay process, but also - alternatively - in a two-step decay of the resonances. The combination of ion-yield measurements with an electron-energy analysis for the $3 d_{5 / 2}^{-1} 5 p$-resonance [14] led to the quantitative decomposition of the total decay of the resonance into a two-step Auger part and a single-step double-ionization part.

In this work we present the first analysis of the fluorescence radiation emitted after the ionization of $3 d$ electrons in krypton just at the ionization thresholds and a few $\mathrm{eV}$ above. Using photon-induced fluorescence spectroscopy (PIFS) we were able to determine cross sections for the total population of excited KrIII and KrIl states. These KrIII states are populated as 
a result of Auger transitions in the first step, whereas the mechanism for the satellite production is unclear.

\section{Experimental procedure}

The experimental set-up has been described previously (see e.g. Ref. [15]). Here we briefly summarize the experimental procedure. Synchrotron radiation in the energy range between 90 and $120 \mathrm{eV}$ from the Berlin electron storage ring BESSY was monochromatized by means of a toroidal-grating monochromator. The monochromator was equipped with a $950 \ell / \mathrm{mm}$ grating. A number of values for the photon bandpass from 190 to $760 \mathrm{meV}$ were used. The monochromatized synchrotron radiation was focussed into an open, differentially pumped target cell with krypton gas at a pressure of $10 \mathrm{~m}$ Torr. The fluorescence radiation emitted by the ions was observed using a $1 \mathrm{~m}$ normal incidence spectrograph (McPherson model 225), in a direction parallel to the vector of the electric field of the exciting radiation. The spectrograph was equipped with a spherical diffraction grating with $1200 \ell / \mathrm{mm}$ and a multiplex detection system [16]. In the present experiment, we observed simultaneously a wavelength range from about 780 to about $1000 \AA$, at a wavelength resolution of $1.8 \AA$. The relative efficiency of the analyzer system (consisting of the diffraction grating and the detector) was estimated on the basis of a new procedure which is described elsewhere in more detail $[17,18]$. In this procedure the well-known fluorescence emission which follows the Auger decay in the ionization continuum of the 3d-electrons in krypton is used for determining the relative spectral sensitivity of the fluorescence analyzer. Fig. 1 shows a fluorescence spectrum recorded after excitation of krypton by $100 \mathrm{eV}$ photons. This spectrum has been corrected for the relative efficiency and the observed spectral lines have been classified in Table 1.

We investigated the energy range from $90 \mathrm{eV}$, i.e. close below the ionization threshold for the $3 \mathrm{~d}_{5 / 2}$ electron, up to $120 \mathrm{eV}$. The fluorescence intensities obtained in these measurements were converted into absolute cross sections by the following procedure: In a first step the cross sections were determined relative to the 4s-photoionization cross section by normalizing the fluorescence intensities to those of the 4s-lines (nos. 1 and 2 in Fig. 1 and Table 1 ) which were mea-

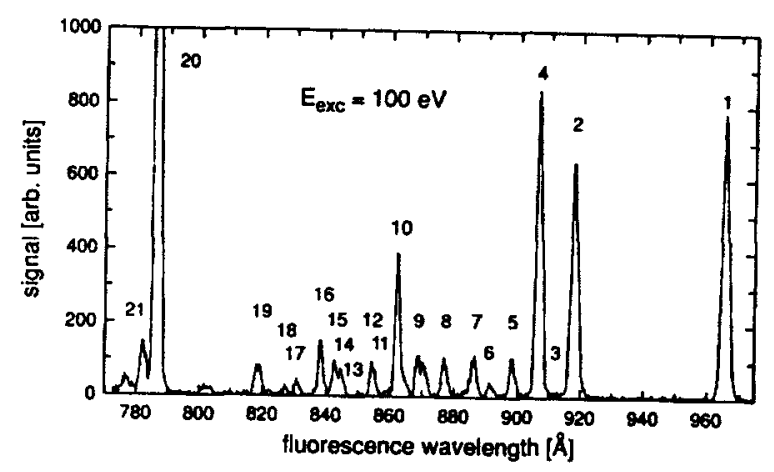

Fig. 1. Fluorescence spectrum recorded at $100 \mathrm{eV}$ excitation energy. The spectral lines observed in the numbered line groups are listed in Table 1 . The spectrum has been corrected for the spectral sensitivity of the detection system.

sured simultaneously. In a second step, we used the value of $0.1 \mathrm{Mb}$ for the 4s-photoionization cross section at $90 \mathrm{eV}$ excitation energy [19] to put the values on the absolute scale. Here it was assumed that the variation of the 4s-photoionization cross section is negligible in the investigated excitation energy range [19].

\section{Results and discussion}

The photoionization cross sections for the different states in KrIII observable in our experiment have been investigated in the energy range beginning just below the $3 \mathrm{~d}_{5 / 2}$-ionization threshold up to $100 \mathrm{eV}$. The cross sections for the population of the states $4 s 4 p^{5}{ }^{3} \mathrm{P}_{J}^{0}$, $4 s 4 p^{5}{ }^{1} P_{1}^{0}$ and $4 s^{0} 4 p^{6}{ }^{1} S_{0}$ in $\mathrm{KrIII}$ are presented in Fig. 2. The energy values for the thresholds stem from Ref. [2], determined by a quantum defect plot. The bandwidth of the exciting synchrotron radiation was $\Delta E=$ $190 \mathrm{meV}$ between the thresholds. The fluorescence intensities for the different $J$-values were summed to obtain the $4 s 4 \mathrm{p}^{53} \mathrm{P}_{J}^{0}$ graph. In the presented energy range the intensity distribution within the $4 s^{2} 4 p^{4}{ }^{3} \mathrm{PJ}_{J}$ $4 s 4 p^{5}{ }^{3} \mathrm{P}_{J}^{0}$ multiplet is constant (population: $51 \%$ of $J=2,37 \%$ of $J=1$ and $12 \%$ of $J=0$ ). This is in accordance with the measured distribution on the $3 \mathrm{~d}_{5 / 2}^{-1} n \mathrm{p}$-resonances and in the ionization continuum [18].

In Fig. 2a a full curve was drawn through the $4 s 4 p^{5}{ }^{1} P_{1}^{0}$ and the $4 s^{0} 4 p^{6}{ }^{1} S_{0}$ data points to guide the eye. It represents the energy dependence of the 
Table 1

Fluorescence transitions observed in the wavelength range from $780 \ddot{A}$ to $1000 \ddot{A}$. The wavelengths stem mainly from Ref. \{23]

\begin{tabular}{|c|c|c|}
\hline & Wavelength $[\ddot{A}$ & Transition \\
\hline 1 & 964.971 & $4 s^{2} 4 p^{5} 2 p_{1 / 2}^{0}-4 s 4 p^{6}{ }^{2} S_{1 / 2}$ \\
\hline 2 & 917.427 & $4 s^{2} 4 p^{5}{ }^{2} p_{3 / 2}^{0}-4 s 4 p^{6}{ }^{2} S_{1 / 2}$ \\
\hline 3 & 911.394 & $4 s^{2} 4 p^{5} P_{1 / 2}^{0}-4 s^{2} 4 p^{4}\left({ }^{3} P\right) 5 s^{4} P_{3 / 2}$ \\
\hline 4 & $907.117^{a}$ & $4 s 4 p^{5}{ }^{1} p_{1}^{0}-4 s^{0} 4 p^{6}{ }^{1} S_{0}$ \\
\hline 5 & 897.806 & $4 s^{2} 4 p^{4}{ }^{3} P_{1}-4 s 4 p^{5}{ }^{3} p_{2}^{0}$ \\
\hline 6 & 891.006 & $4 s^{2} 4 p^{5}{ }^{2} P_{1 / 2}^{0}-4 s^{2} 4 p^{4}\left({ }^{3} P\right) 5 s^{4} P_{1 / 2}$ \\
\hline 7 & 886.300 & $4 s^{2} 4 p^{5}{ }^{2} p_{3 / 2}^{0}-4 s^{2} 4 p^{4}\left({ }^{3} p\right) 5 s^{4} P_{5 / 2}$ \\
\hline 7 & 884.141 & $4 s^{2} 4 p^{5}{ }^{2} P_{1 / 2}^{0}-4 s^{2} 4 p^{4}\left({ }^{3} P\right) 5 s^{2} P_{3 / 2}$ \\
\hline 8 & 876.676 & $4 s^{2} 4 p^{4}{ }^{3} P_{0}-4 s 4 p^{5}{ }^{3} P_{1}^{o}$ \\
\hline 9 & 870.842 & $4 s^{2} 4 p^{4}{ }^{3} P_{1}-4 s 4 p^{5} P_{1}^{0}$ \\
\hline 9 & 868.871 & $4 s^{2} 4 p^{5}{ }^{2} P_{3 / 2}^{0}-4 s^{2} 4 p^{4}\left({ }^{3} P\right) 5 s^{4} P_{3 / 2}$ \\
\hline 10 & 864.821 & $4 s^{2} 4 p^{5}{ }^{2} p_{1 / 2}-4 s^{2} 4 p^{4}\left({ }^{3} P\right) 4 d^{4} D_{3 / 2}$ \\
\hline 10 & 862.582 & $4 s^{2} 4 p^{4}{ }^{3} p_{2}-4 s 4 p^{5}{ }^{3} p_{2}^{0}$ \\
\hline 11 & 859.037 & $4 s^{2} 4 p^{5}{ }^{2} P_{1 / 2}^{0}-4 s^{2} 4 p^{4}\left({ }^{3} P\right) 4 d^{4} D_{1 / 2}$ \\
\hline 12 & 854.73 & $4 s^{2} 4 p^{4}{ }^{3} p_{1}-4 s 4 p^{5}{ }^{3} p_{0}^{0}$ \\
\hline 13 & 850.319 & $4 s^{2} 4 p^{52} P_{3 / 2}^{0}-4 s^{2} 4 p^{4}\left({ }^{3} P\right) 5 s^{4} P_{1 / 2}$ \\
\hline 14 & 844.064 & $4 s^{2} 4 p^{5}{ }^{2} p_{3 / 2}^{0}-4 s^{2} 4 p^{4}\left({ }^{3} P\right) 5 s^{2} P_{3 / 2}$ \\
\hline 15 & $842.04^{b}$ & $4 s^{2} 4 p^{3}{ }^{4} S_{3 / 2}^{o}-4 s 4 p^{4}{ }^{4} P_{s / 2}$ \\
\hline 16 & 837.662 & $4 s^{2} 4 p^{4}{ }^{3} p_{2}-4 s 4 p^{5}{ }^{3} p_{1}$ \\
\hline 17 & 830.375 & $4 s^{2} 4 p^{5}{ }^{2} P_{3 / 2}^{o}-4 s^{2} 4 p^{4}\left({ }^{3} P\right) 4 d^{4} D_{5 / 2}$ \\
\hline 18 & 826.434 & $4 s^{2} 4 p^{5}{ }^{2} P_{3 / 2}^{0}-4 s^{2} 4 p^{4}\left({ }^{3} P\right) 5 s^{2} P_{1 / 2}$ \\
\hline 19 & 821.154 & $4 s^{2} 4 p^{5}{ }^{2} P_{3 / 2}^{0}-4 s^{2} 4 p^{4}\left({ }^{3} P\right) 4 d^{4} D_{1 / 2}$ \\
\hline 19 & 818.149 & $4 s^{2} 4 p^{5}{ }^{2} P_{1 / 2}^{0}-4 s^{2} 4 p^{4}\left({ }^{1} D\right) 5 s^{2} D_{3 / 2}$ \\
\hline 19 & 816.82 & $4 s^{2} 4 p^{34} S_{3 / 2}^{o}-4 s 4 p^{4}{ }^{4} P_{3 / 2}$ \\
\hline 20 & 785.968 & $4 s^{2} 4 p^{4}{ }^{1} D_{2}-4 s 4 p^{5}{ }^{1} p_{1}^{\circ}$ \\
\hline 21 & 783.724 & $4 s^{2} 4 p^{5}{ }^{2} P_{1 / 2}^{0}-4 s^{2} 4 p^{4}\left({ }^{3} P\right) 4 d^{4} P_{3 / 2}$ \\
\hline 21 & 782.096 & $4 s^{2} 4 p^{5}{ }^{2} p_{3 / 2}^{0}-4 s^{2} 4 p^{4}\left({ }^{1} D\right) 5 s^{2} D_{5 / 2}$ \\
\hline 21 & 781.58 & $4 s^{2} 4 p^{3}{ }^{2} D_{5 / 2}-4 s 4 p^{4}{ }^{2} D_{5 / 2}$ \\
\hline
\end{tabular}

a From Ref. [24]. b From Ref. [25].

$3 d_{5 / 2}$ and $3 d_{3 / 2}$-ionization cross sections near their threshold energies, since only a negligible population of especially the $4 s^{0} 4 \mathrm{p}^{6}{ }^{1} \mathrm{~S}_{0}$ state was observed on the $3 d^{-1} n p$-resonances [18]. Note that for the $4 s 4 p^{5} P_{i}$ state (Fig. $2 a$ ) the $3 d_{3 / 2}^{-1} 6$ p-resonance would lie near the energy of the $3 d_{5 / 2}$ threshold.

The cross section for the population of the $4 s 4 p^{53} \mathrm{PJ}$ states in Krlll is nearly constant (see Fig. 2b) with a strong enhancement observed at the $3 \mathrm{~d}_{3 / 2}^{-1} 6 \mathrm{p}$ resonance near the $3 \mathrm{~d}_{5 / 2}$-ionization threshold. This enhancement exceeds that of the ${ }^{1} P$ state.

For an excitation energy larger than $96.0 \mathrm{eV}$, the
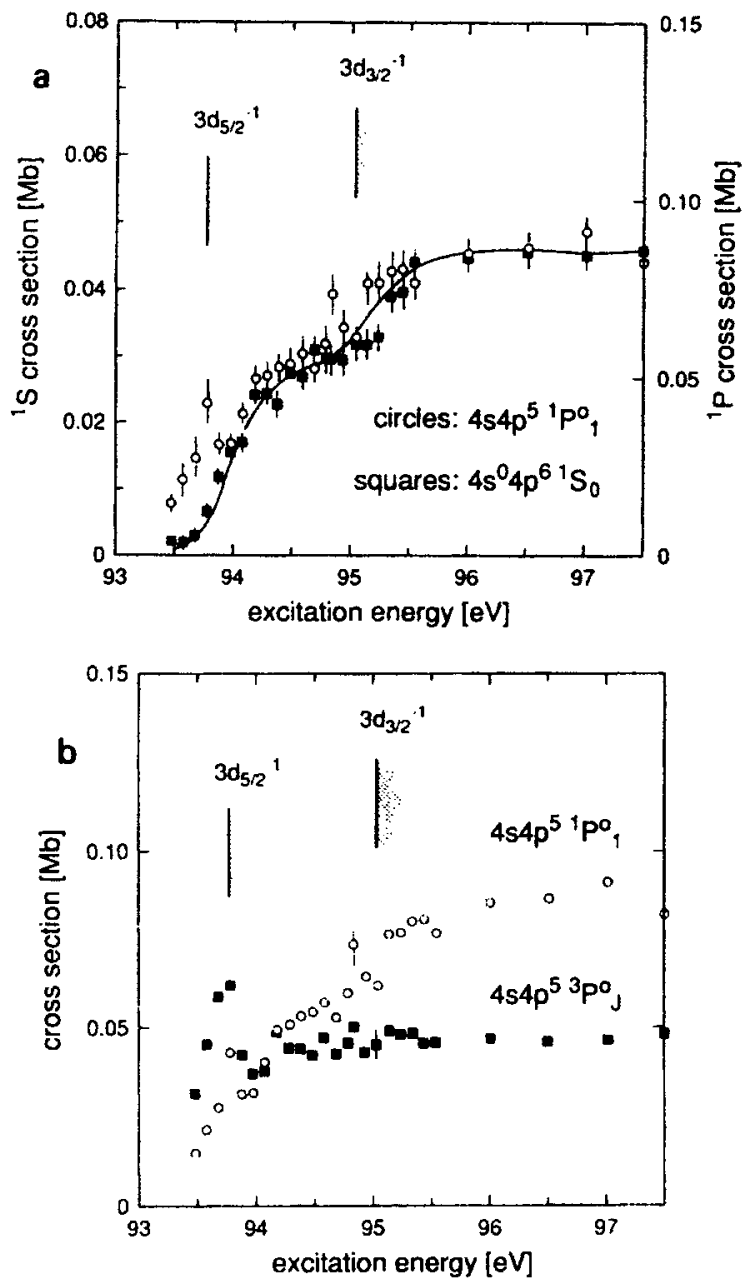

Fig. 2. Cross sections for production of the KrIll states $4 s 4 p^{51} P_{1}^{0}$ and $4 s^{0} 4 p^{6} i S_{0}$ (a) and for $4 s 4 p^{53} P_{j}^{0}$ and $4 s 4 p^{5}{ }^{1} P_{1}^{\circ}(b)$ in the $3 d-1$ hreshold region. The scale for the ${ }^{\prime} S_{0}$ state is given on the left-hand side of $(a)$, the scale for the ${ }^{1} \mathrm{P}_{1}^{\circ}$ state is given on the right. The shape of the 3d-ionization cross section curve is assumed to be represented approximately by the full curve in (a).

observed states in KrIII are populated at the same ratio as observed for the Auger decay which follows 3d-ionization (100:54:52 for ${ }^{1} \mathrm{P}_{1}^{0.3} \mathrm{P}_{J}^{0}: \mathrm{S}_{0}[20]$ ). In the energy range from 96.0 up to $100.0 \mathrm{eV}$, the fluorescence spectra were recorded with a primary bandwidth of $\Delta E=760 \mathrm{meV}$ and in steps of $500 \mathrm{meV}$. The signal belonging to the population of the states in $\mathrm{Krll}$ is relatively weak in the threshold energy region (see below). 


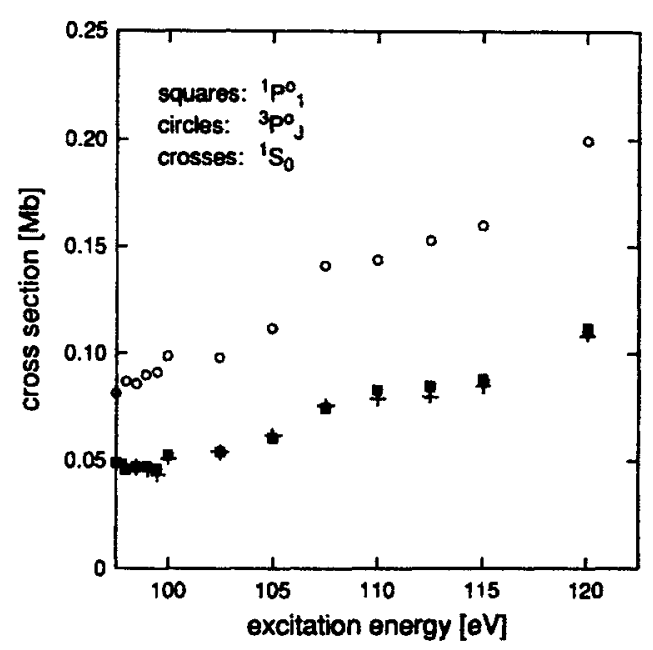

Fig. 3. Cross sections for the KrIll states $4 s 4 p^{51} p_{1}$, $4 s 4 p^{53} \mathrm{p}_{j}^{0}$ and $4 s^{0} 4 \mathrm{p}^{6}{ }^{1} \mathrm{~S}_{0}$ up to $120 \mathrm{eV}$ excitation energy.

In the energy region from 100 up to $120 \mathrm{eV}$, fluorescence spectra were recorded with a bandwidth of $\Delta E=400 \mathrm{meV}$ and a stepwidth of $2.5 \mathrm{eV}$. Fig. 3 shows the photoionization cross sections for the observed states in KrIII from 97.5 up to $120 \mathrm{eV}$ excitation energy. In this energy range, the intensity distributions in the ${ }^{3} \mathrm{P}_{j}^{0}$ multiplet as well as in the other observed multiplets are constant within the statistical errors. Assuming a value of $\sigma_{3 d}(100 \mathrm{eV})$ $=0.78 \mathrm{Mb}[21]$, the observed cross sections correspond to the following fractions of the total decay of the 3d-hole: $(12.7 \pm 1.5) \%$ for the decay ending in the $4 \mathrm{~s} 4 \mathrm{p}^{51} \mathrm{P}_{\mathrm{i}}^{0}$ state and $(6.8 \pm 0.9) \%$ for $4 \mathrm{~s} 4 \mathrm{p}^{53} \mathrm{P}_{\mathrm{J}}^{0}$ and $(6.5 \pm 0.8) \%$ for $4 s^{0} 4 \mathrm{p}^{6}{ }^{1} \mathrm{~S}_{0}$, respectively. Taking a value of $\sigma_{3 \mathrm{~d}}(120 \mathrm{eV})=1.98 \mathrm{Mb}$ [22], fractions of $(10.1 \pm 1.2) \%\left({ }^{1} \mathrm{P}_{1}^{\circ}\right),(5.7 \pm 0.8) \%\left({ }^{3} \mathrm{P}_{j}^{0}\right)$ and $(5.5$ $\pm 0.7) \%\left({ }^{1} S_{0}\right)$ result.

In Fig. 4, the photoionization cross sections for the states $4 s^{2} 4 \mathrm{p}^{4}\left({ }^{3} \mathrm{P}\right) 5 \mathrm{~s}^{4} \mathrm{P}_{J}\left(J=\frac{5}{2}, \frac{3}{2}, \frac{1}{2}\right)$, $4 s^{2} 4 \mathrm{p}^{4}\left({ }^{3} \mathrm{P}\right) 5 \mathrm{~s}^{2} \mathrm{P}_{J}\left(J=\frac{3}{2}, \frac{1}{2}\right)$ and $4 \mathrm{~s}^{2} 4 \mathrm{p}^{4}\left({ }^{2} \mathrm{P}\right) 4 \mathrm{~d}^{4} \mathrm{D}_{J}$ $\left(J=\frac{5}{2}, \frac{3}{2}, \frac{1}{2}\right)$ are shown as a function of primary photon energy, with the signals of the individual multiplet components summed. Their intensity ratios, which are constant in the investigated energy range, are listed in Table 2. The sum of the cross sections for the population of the observed states in KrII amounts to $3.5 \%$ of the $3 \mathrm{~d}$-cross section [21]. A reference spectrum at $90 \mathrm{eV}$ excitation energy was recorded and the relative population probabilities at this energy have
Table 2

Relative population probabilities for $\mathrm{KrII}$ satellite states at $90 \mathrm{eV}$ excitation energy and in the ionization continuum for the 3d-electrons

\begin{tabular}{|c|c|c|}
\hline Ionic state & $90 \mathrm{eV}$ & $\begin{array}{l}\text { Ionization continuum } \\
(100-120 \mathrm{eV})\end{array}$ \\
\hline $4 s^{2} 4 p^{4}\left({ }^{3} P\right) 5 s^{4} P_{5 / 2}$ & 100 & 100 \\
\hline $4 s^{2} 4 p^{4}\left({ }^{3} P\right) 5 s^{4} P_{3 / 2}$ & 145 & 84 \\
\hline $4 s^{2} 4 p^{4}\left({ }^{3} P\right) 5 s^{4} P_{1 / 2}$ & 84 & 43 \\
\hline $4 s^{2} 4 p^{4}\left({ }^{3} P\right) 5 s^{2} P_{3 / 2}$ & 114 & 59 \\
\hline $4 s^{2} 4 p^{4}\left({ }^{3} P\right) 5 s^{2} P_{1 / 2}$ & 44 & 13 \\
\hline $4 s^{2} 4 p^{4}\left({ }^{3} P\right) 4 d^{4} D_{s / 2}$ & 25 & 28 \\
\hline $4 s^{2} 4 p^{4}\left({ }^{3} P\right) 4 d^{4} D_{3 / 2}$ & 89 & 47 \\
\hline $4 s^{2} 4 p^{4}\left({ }^{3} P\right) 4 d^{4} D_{1 / 2}$ & 14 & 7 \\
\hline
\end{tabular}

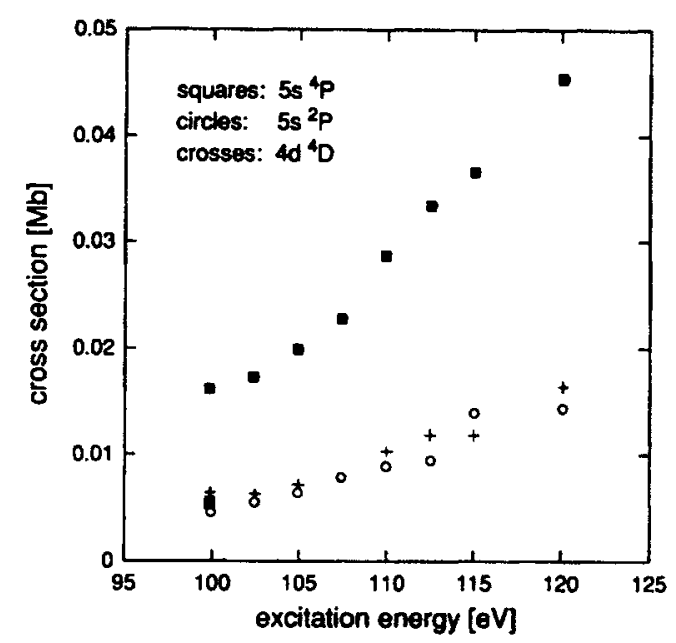

Fig. 4. Cross sections for the KrII states $4 s^{2} 4 p^{4} 5 s{ }^{4} P_{J}$, $4 s^{2} 4 p^{4} 5 s^{2} P_{J}$ and $4 s^{2} 4 p^{4} 4 d^{4} D$, up to $120 \mathrm{eV}$ excitation energy.

been included in Table 2 . The cross section for the $4 s^{2} 4 p^{4}\left({ }^{3} P\right) 5 s^{4} P_{5 / 2}$ state at $90 \mathrm{eV}$ was found to be $(18$ $\pm 2) \%$ of that at $100 \mathrm{eV}$. The cross sections increased by a factor of 2.8 from 100 up to $120 \mathrm{eV}$. A similar increase has been observed for the 3d-photoionization cross section $[21,22]$. This correspondence is not yet understood. Since cascades from $5 p-5 s$ and $5 p-4 d$ transitions in KrII are included in the measured cross sections, also $4 p^{4} 5 p$ satellites have to be considered in the discussion of the unexplained energy dependence. Preliminary theoretical investigations [26] show that the energy dependence cannot be due to a simple final-state configuration interaction with the $3 \mathrm{~d}^{-1} \varepsilon f$ continuum. They also show that the cross sec- 
tions for direct $4 s$-satellite production are negligible in comparison with the measured cross sections.

The measured energy dependence for the population of the observed satellite states in KrIl should motivate theoretical investigations of many-body effects which have not been included so far in the calculations of the 3d-photoionization cross section and of cross sections for satellite production.

\section{Acknowledgement}

The authors like to thank Professor V.L. Sukhorukov and Dr. B.M. Lagutin for helpful discussions. This work was funded by the German Federal Minister for Research and Technology under contract nos. 05 5UKAXB and 05 5RGAXB.

\section{References}

[1] Th.M. El-Sherbini and M.J. van der Wiel, Physica 62 (1972) 119.

[2] G.C. King, M. Tronc, F.H. Read and R.C. Bradford, J. Phys. B 10 (1977) 2479.

[3] T. Hayaishi, Y. Morioka. Y. Kageyama, M. Watanabe, I.H. Suzuki, A. Mikumi, G. Isoyama, S. Asaoka and M. Nakamura, J. Phys. B 17 (1984) 3511.

[4] W. Eberhardt, G. Kalkoffen and C. Kunz, Phys. Rev. Lett. 4 (1978) 156.

[5] H. Aksela, S. Aksela, H. Pulkkinen, G.H. Bancroft and K.H. Tan, Phys. Rev. A 33 (1986) 3876.

[6] D.W. Lindle, P.A. Heimann, T.A. Ferrett, M.N. Piancastelli and D.A. Shirley, Phys. Rev. A 35 (1987) 4605.

[7] P.A. Heimann, D.W. Lindle, T.A. Ferrett, S.H. Lin, L.J. Medhurst, M.N. Piancastelli, D.A. Shirley, U. Becker, H.G. Kerkhoff, B. Langer, D. Szostak and R. Wehlitz, J. Phys. B 20 (1987) 5005.

[8] T.A. Carlson, D.R. Mullins, C.E. Beall, B.W. Yates, J.W. Taylor, D.W. Lindle, B.P. Pullen and F.A. Grimm, Phys. Rev. Lett. 60 (1988) 1382.
[9] T.A. Carlson, D.R. Mullins, C.E. Beall, B.W. Yates. J.W. Taylor, D.W. Lindle, B.P. Pullen and F.A. Grimm, Phys. Rev. A 39 (1989) 1170.

[10] H. Aksela, S. Akseia, H. Pulkkinen and A. Yagishita. Phys. Rev. A 40 (1989) 6275.

[11] H. Aksela, S. Aksela, A. Kivimäki and O.-P. Sairanen. Phys. Scr. 41 (1990)425.

[12] E. van Raven, M. Meyer, M. Pahler and B. Sonntag, J. Electron Spectrosc. Related Phenom. 52 (1990) 677.

[13] T. Hayaishi, A. Yagishita, E. Murakami, E. Shigesama, Y. Morioka and T. Sasaki, J. Phys. B 23 (1990) 1633.

[14] P. Lablanquie and P. Morin. J. Phys. B 24 (1991) 4349.

[15] K.-H. Schartner, P. Lenz, B. Möbus, B. Magel, H. Schmoranzer and M. Wildberger, Phys. Scr. 41 (1990) 853 , and references therein.

[16] B. Kraus, K.-H. Schartner, F. Folkmann, A.E. Livingston and P.H. Mokler, in: EUV, X-ray, and gamma-ray instrumentation for astronomy and atomic physics, eds. G.J. Hailey and O.H.W. Siegmund (Bellingham, 1989) p. 217.

[17] K.-H. Schartner, B. Möbus, M. Wildberger, A. Ehresmann and $H$. Schmoranzer, BESSY Annual Report 1991, p. 514.

[18] M. Wildberger, Ph.D. thesis, Universität Kaiserslautern, Kaiserslautern (1993).

[19] A. Ehresmann, F. Vollweiler, H. Schmoranzer, V.L. Sukhorukov, B.M. Lagutin, I.D. Petrov, G. Mentzel and K.-H. Schartner, J. Phys. B 27 (1994) 1489.

[20] L.O. Werme. T. Bergmark and K. Siegbahn, Phys. Scr. 6 (1972) 141.

[21] E. Murakami, T. Hayaishi, A. Yagishita and Y. Morioka, Phys. Scr. 41 (1990) 468.

[22] D.W. Lindle, P.A. Heimann, T.A. Ferrett, P.H. Kobrin, C.M. Truesdale, U. Becker, H.G. Kerkhoff and D.A. Shirley, Phys. Rev. A 33 (1986) 319.

[23] R.L. Kelly, Atomic and ionic spectrum lines below $2000 \AA$. ORNL-5922 (1982).

[24] M. Agentoft, T. Andersen, J.E. Hansen, W. Persson and S.-G. Pettersson, Phys. Scr. 29 (1984) 57.

[25] B.C. Fawcett and G.E. Bromage, J. Phys. B 13 (1980) 2711.

[26] B.M. Lagutin and V.L. Sukhorukov, private communication (1994). 